\title{
Acciones entrelazadas contra daños ambientales graves: el impacto de Shell en Nigeria
}

\section{Intertwined Actions against serious environmental damage: The impact of Shell in Nigeria}

\section{ANTONI PIGRAU \& ANTONIO CARDESA-SALZMANN*}

Resumen: La presión de la demanda de recursos naturales y de energía en el mundo desarrollado sigue creciendo y, con ello, los impactos negativos sobre el medio ambiente y los derechos de las personas vinculados en especial a las actividades extractivas llevadas a cabo por las mayores empresas transnacionales. La suma de tres factores - la vinculación entre daños al medio ambiente y vulneración de derechos humanos, la debilidad de la legislación y la incapacidad o la falta de voluntad de la institucionalidad que debe aplicarla en muchos de los países donde se desarrollan dichas actividades y la inexistencia de vías internacionales para reclamar la responsabilidad directamente a las empresas - ha llevado a las víctimas de graves daños ambientales a utilizar a la vez todo tipo de vías disponibles (judiciales y no judiciales, nacionales e internacionales), en lo que cabe denominar acciones entrelazadas, puesto que todas ellas acaban por reforzarse mutuamente y aumentan la visibilidad del problema. El objetivo de este trabajo es mostrar esta realidad a partir del caso concreto del impacto de las actividades de la empresa petrolera Shell, una de las empresas que opera en el delta del Níger.

Palabras clave: empresas transnacionales - responsabilidad - daños ambientales - justicia ambiental

Summary: The demand for natural resources and energy continues to grow in the developed world and, with it, the negative impacts on the environment and the rights of the peoples directly affected by extractive activities undertaken by the largest transnational corporations. Three factors compel victims of serious environmental damage to resort simultaneously to any available avenue of legal action (whether judicial or non-judicial, national or international): (1) the link between environmental damage and human rights abuses; (2) weak legislation, combined with the inability or unwillingness

* Este artículo se basa en uno de los estudios de caso realizados en el marco de un proyecto más amplio de investigación vinculado al Proyecto Environmental Justice Organizations, Liabilities and Trade (EJOLT), VII Programa Marco de la Unión Europea, Número FP7-SCIENCE-IN-SOCIETY-2010-1, y al proyecto de investigación, financiado por el Ministerio de Ciencia e Innovación de España, «La garantía jurídica de la vertiente intrageneracional de la justicia ambiental como aspecto social del desarrollo sostenible» (DER2010-19529).

Antoni Pigrau es doctor en Derecho por la Universidad de Barcelona. Catedrático de Derecho Internacional Público y director del Centro de Estudios de Derecho Ambiental de Tarragona (CEDAT, www.cedat.cat) de la Universidad Rovira i Virgili, Tarragona, España. Correo electrónico: antoni. pigrau@urv.cat.

Antonio Cardesa-Salzmann es doctor en Derecho por la Universidad de Barcelona. Es investigador del CEDAT en la Universidad Rovira i Virgili. Correo electrónico: antonio.cardesa@urv.cat 
of the law-enforcing institutions in many countries where such extractive activities take place; and (3) the absence of international avenues to claim direct liability from corporations. We call this phenomenon intertwined actions, as the various judicial actions undertaken by the victims end up mutually reinforcing and increasing the visibility of the problem. The aim of this paper is to show this reality on the basis of the various instances of litigation that are taking place in the specific context of Shell's activities in the Niger delta.

Key words: transnational corporations - liability - environmental damages environmental justice

CONTENIDO: I. ANTECEDENTES.- II. ACCIONES EMPRENDIDAS ANTE ORGANISMOSYTRIBUNALES NACIONALES.- II.1. NIGERIA.- II.2. PAÍSES BAJOS.II.2.1. ORUMA, GOI, IKOT ADA UDO.- II.2.2. PUNTO NACIONAL DE CONTACTO DE LA OCDE.- II.3. REINO UNIDO. - II.4.ESTADOS UNIDOS.- II.4.1. WIWA V. SHELL.II.4.2. KIOBEL V. SHELL.- III. ACCIONES EMPRENDIDAS ANTE INSTITUCIONES INTERNACIONALES.- III.1 COMISIÓN AFRICANA DE DERECHOS HUMANOS Y DE LOS PUEBLOS. - III.2. CORTE DE JUSTICIA DE LA COMUNIDAD ECONÓMICA DE ESTADOS DE ÁFRICA OCCIDENTAL.- IV. CONCLUSIONES.

\section{ANTECEDENTES}

El delta del Níger es uno de los humedales y de los ecosistemas marinos más importantes del mundo y comprende un área de unos 70000 kilómetros cuadrados. Nigeria es el primer productor de petróleo en África ${ }^{1}$. La extracción de petróleo por parte de diversas empresas ha tenido un impacto devastador en la región, producido por las propias operaciones extractivas, por la práctica ininterrumpida de quemar los gases procedentes de las operaciones de extracción de petróleo, por los continuos derrames de petróleo y por la deforestación, destruyendo el suelo, los recursos naturales y la economía de sus comunidades, basada en la agricultura de subsistencia y la pesca.

Según un informe del Programa de las Naciones Unidas para el Medio Ambiente (PNUMA) presentado en agosto de 20112, la región es la zona más contaminada del mundo: en el delta del Níger se han vertido 2100 millones de litros de crudo en las cinco décadas en las que se ha extraído petróleo, a un ritmo de 42 millones de litros derramados cada año (cifra prácticamente idéntica a la del derrame causado en 1989 por el petrolero Exxon Valdez en Alaska). La recuperación total de la región requeriría entre veinticinco y treinta años de trabajo con un coste

1 Ver el informe de la US Energy Information Administration (EIA) sobre Nigeria, (http://www.eia.gov/ countries/analysisbriefs/Nigeria/nigeria.pdf), 2012.

2 United Nations Environment Programme (UNEP). Environmental Assessment of Ogoniland Report. Nairobi: 2011, (http://www.unep.org/nigeria/). El Informe fue encargado por el Gobierno de Nigeria y recibió financiación de Shell, por lo que se basa en datos de la propia empresa y no entra en el ámbito de las responsabilidades. 
estimado solamente para los primeros cinco años de mil millones de dólares ${ }^{3}$. Y las personas han tenido que convivir con la contaminación, en muchos casos a lo largo de toda su vida. Se ha denunciado que las empresas petroleras operan en Nigeria por debajo de los estándares internacionales de buenas prácticas en el sector ${ }^{4}$.

La empresa Royal Dutch-Shell empezó a operar en Nigeria en 1957. En la actualidad Shell Petroleum Development Company of Nigeria (SPDC) es el operador de un acuerdo de joint venture que implica a la Nigerian National Petroleum Corporation (NNPC), que posee el 55 por ciento, Shell (30\%), Elf Petroleum Nigeria Ltd. (10\%) y Agip Nigeria Plc. (5\%) y que ocupa más de 30000 kilómetros cuadrados. Durante estos años, la empresa ha mantenido una estrecha relación con los distintos gobiernos de Nigeria -muchos de ellos gobiernos militares surgidos de golpes de Estado- y ha colaborado con ellos para reprimir la oposición popular a la continuación de sus operaciones en la región, facilitando sus infraestructuras y apoyo logístico y financiando a las tropas gubernamentales 5 . La represión fue especialmente cruel durante la década de 1990 contra el pueblo ogoni — que habita la región del mismo nombre, en el estado de Rivers ${ }^{6}$ - y la organización que lideraba la oposición, el Movimiento para la Supervivencia del Pueblo Ogoni (MOSOP). Shell suspendió sus actividades en la zona en 1993. En 1994, junto a la destrucción de decenas de pueblos y aldeas, detenciones masivas, más de cien mil desplazados internos y una cifra estimada de dos mil civiles muertos, nueve miembros del MOSOP fueron detenidos, puestos en régimen de incomunicación bajo custodia militar, y luego juzgados sumariamente, condenados por asesinato y ejecutados en 1995 en la horca. Por todo ello han entrado en juego normas fundamentales del derecho internacional de los derechos humanos, como las que prohíben la tortura, las detenciones arbitrarias o los crímenes contra la humanidad. Tales normas forman parte del derecho internacional consuetudinario o están recogidas en distintos tratados internacionales, como la Convención contra la Tortura y Otros Tratos o Penas Crueles, Inhumanos o Degradantes, de 1984, el Pacto Internacional de Derechos Civiles y Políticos de $1966^{8}$ o la Carta

3 Ibídem, pp. 226-227.

4 Ver SteINER, Richard. Double Standard. Shell Practices in Nigeria Compared With International Standards to Prevent and Control Pipeline Oil Spills and the Deepwater Horizon Oil Spill, (http://www. foei.org/en/resources/publications/pdfs/2010/double-standard-shell-practices-in-nigeria-comparedwith-international-standards/view), 2010.

5 Ver HumAn RightS WATCH. «The Price of Oil: Corporate Responsibility and Human Rights Violations in Nigeria's Oil Producing Communities», HRW Index No. 1-56432-225-4, 1999, (http://www.hrw.org/ reports/1999/nigeria/).

6 La región de Ogoni posee diversos recursos naturales muy valiosos, como el tercer bosque de manglares más grande del mundo y uno de los mayores bosques lluviosos que quedan en Nigeria.

7 Adoptada por la Asamblea General de las Naciones Unidas en su resolución 39/46, de 10 de diciembre de 1984, (http://www2.ohchr.org/spanish/law/cat.htm).

8 Adoptado por la Asamblea General de las Naciones Unidas en su resolución 2200 A (XXI), de 16 de diciembre de 1966, (http://www2.ohchr.org/spanish/law/ccpr.htm).

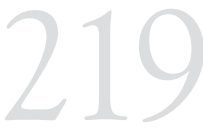

ACCIONES

ENTRELAZADAS

CONTRA DAÑOS

AMBIENTALES

GRAVES: EL

IMPACTO DE

SHELL EN NIGERIA

INTERTWINED

ACTIONS

AGAINST SERIOUS ENVIRONMENTAL

DAMAGE:THE

IMPACT OF SHELL

IN NIGERIA 
Africana de Derechos Humanos y de los Pueblos de 27 de junio de $1981^{9}$.

La historia de la explotación del petróleo en Nigeria ha estado plagada de incidentes de contaminación. Unos de los últimos episodios de mayor gravedad se produjo el 20 de diciembre de 2011 con el vertido de cuarenta mil barriles de petróleo de la plataforma marina del yacimiento de Bonga, cercana a las costas del estado de Bayelsa.

Los afectados por esta situación han utilizado un conjunto de vías distintas, judiciales y no judiciales: no solo ante las instituciones de Nigeria sino también ante los tribunales de los Países Bajos (donde reside la empresa matriz) y de los Estados Unidos (utilizando la jurisdicción extraterritorial civil), ante los órganos del sistema africano de protección de los derechos humanos, ante el Tribunal de Justicia de la Comunidad Económica de Estados de África Occidental y ante los Puntos Nacionales de Contacto (PNC) de la Organización para la Cooperación y el Desarrollo Económico (OCDE) en los Países Bajos y en el Reino Unido. En este trabajo se denomina acciones entrelazadas a esta realidad en la que se promueven acciones diversas, de carácter judicial y no judicial, ante instituciones nacionales, en distintos países, e internacionales, y relativas a diversos aspectos concretos de un mismo problema de fondo, que se manifiesta claramente en el caso del impacto de Shell en Nigeria, pero que puede constatarse también en otros $\operatorname{casos}^{10}$.

\section{ACCIONES EMPRENDIDAS ANTE ORGANISMOS Y TRIBUNALES NACIONALES}

\section{II.1. Nigeria}

La legislación nigeriana prevé la responsabilidad de las empresas por los derrames causados y la obligación de indemnizar a los perjudicados. Así, la Nigerian Federal Environmental Protection Agency Act de 1988 establece que, después de un derrame de petróleo, las empresas deben «begin immediate clean-up operations following the best available clean-up practice and removal methods» La Oil Pipelines Act de 1990, en su apartado 11.5.c, dispone que las empresas deben pagar una compensación «to any person suffering damage (other than on account of his own default or on account of the malicious act of a third person) as a consequence of any breakage of or leakage from the pipeline or an ancillary installation, for any such damage not otherwise made good» ${ }^{11}$.

9 Organization of African Unity. African Charter on Human and Peoples' Rights ("Banjul Charter»), 27 de junio de 1981, CAB/LEG/67/3 rev. 5, 21 I.L.M. 58 (1982), (http://www.unhcr.org/refworld/ docid/3ae6b3630.html).

10 Ver PIGRAU, Antoni \& otros. Legal avenues for EJOs to claim environmental liability. EJOLT Report 4, (http://www.ejolt.org/section/resources/reports/), 2012.

11 Oil Pipelines Act, Chapter 338, Laws of the Federation of Nigeria, (http://www.nigeria-law.org/Oil\%20 Pipelines\%20Act.htm), 1990. 
Y la Nigerian Petroleum Act de 1969 establece en su sección 8.1.g que el Ministro «may direct in writing the suspension of any operations which in his opinion are not being conducted in accordance with good oil field practice» ${ }^{12}$.

Cientos de demandas en relación con derrames de petróleo se han presentado contra Shell y otras empresas petroleras ante los tribunales de Nigeria, pero con frecuencia las decisiones se han demorado años y son pocas las decisiones judiciales firmes, que han comportado algunas compensaciones económicas, pero raras veces la restauración del medio ambiente $^{13}$.

Uno de los casos más relevantes se inició cuando, el 20 de junio de 2005, un representante de la comunidad de Iwherekan, en el delta del Níger, presentó una demanda de protección de derechos fundamentales contra el Gobierno de Nigeria y Shell por la utilización de la técnica de quema permanente en la atmósfera de gas (gas flaring), para separarlo del petróleo. Ello genera impactos negativos sobre la salud de las personas, incrementa las emisiones de gases de efecto invernadero, contamina los alimentos y el agua, reduce las cosechas y causa lluvia ácida. El 14 de noviembre de 2005 la Alta Corte Federal de Nigeria declaró que tal práctica era inconstitucional ${ }^{14}$ y que las empresas petroleras tenían que cesar la quema de gas en el delta del Níger ${ }^{15}$. Shell apeló esta decisión. El 16 de diciembre de 2005 se presentó una nueva demanda contra Shell y otras empresas por no haber cesado la quema de gas. La Corte Suprema de Nigeria decidió en abril de 2006 que Shell tenía que detener la quema de gas en el plazo de un año en la comunidad de Iwherekan. En este caso se basa la adopción de la Gas Flaring Prohibition and Punishment Bill, el 2 de julio de 2009. Pero la entrada en vigor de la prohibición completa se ha ido retrasando en diversas ocasiones. Y la reciente Petroleum Industry Bill de 2012 parece dar cobertura a nuevas excepciones.

En otro litigio, el 5 de julio de 2010, tras casi diez años desde su inicio, el Tribunal Federal de Nigeria condenó a Shell Nigeria a pagar unos 100

12 Petroleum Act. Cap. 350 L.F.N. 1990 Act Cap. 10 L.F.N. 20041, (http://www.babalakinandco.com/ resources/lawsnigeria/LAWS/90350petroleum\%20act.htm).

13 Ver FRYNAS, Jedrzej George. "Social and environmental litigation against transnational firms in Africa», Journal of Modern African Studies, 42, 3 (2004), pp. 363-388.

14 «That section 3(2)(a) and (b) of the Associated Gas Re-Injection Act and section 1 of the Associated Gas Re-Injection (Continued Flaring of Gas) Regulations section 1.43 of 1984, under which gas flaring in Nigeria may be allowed are inconsistent with the applicant's rights to life and/or dignity of human person enshrined in sections 33(1) and 34(1) of the Constitution of the Federal Republic of Nigeria, 1999 and articles 4, 16 and 24 of the African Charter on Human and Peoples' Rights (Ratification and Enforcement) Act, cap A9, vol 1, Laws of the Federation of Nigeria, 2004) and are therefore unconstitutional, null and void by virtue of section 1(3) of the same Constitution" (Gbemre $v$ Shell Petroleum Development Company Nigeria Limited and Others (2005) AHRLR 151 (NgHC 2005)).

15 «that the 1st and 2nd respondents [Shell Petroleum Development Company Nigeria Ltd., Nigerian National Petroleum Corporation] are accordingly restrained whether by themselves, their servants or workers or otherwise from further flaring of gas in applicants' community and are to take immediate steps to stop the further flaring of gas in the applicant's community" (ibídem).

ACCIONES

ENTRELAZADAS

CONTRA DAÑOS

AMBIENTALES

GRAVES: EL

IMPACTO DE

SHELL EN NIGERIA

INTERTWINED

ACTIONS

AGAINST SERIOUS ENVIRONMENTAL

DAMAGE:THE

IMPACT OF SHELL

IN NIGERIA 
millones de dólares en concepto de daños y perjuicios a otra comunidad, la de Ejama-Ebubu, por un vertido de petróleo sucedido cuarenta años atrás, en 1970, que afectó a unas 250000 hectáreas. La indemnización incluye daños basados en el valor de los cultivos, la pérdida de ingresos derivados de la agricultura y la caza, el suministro de agua, los riesgos para la salud, el impacto psicológico y la profanación de los santuarios, entre otros elementos. Además el juez dispuso que Shell Nigeria tenía que descontaminar y rehabilitar la zona hasta dejarla tal como estaba antes del vertido.

En todo caso, es un hecho que la litigación ha pasado a formar parte de las estrategias de las organizaciones que defienden la justicia ambiental en Nigeria ${ }^{16}$.

\section{II.2. Países Bajos}

\section{II.2.1. Oruma, Goi, Ikot Ada Udo}

El 9 de mayo de 2008 campesinos y pescadores residentes en distintas aldeas del delta del Níger (Oruma, Goi y Ikot Ada Udo), apoyados por Amigos de la Tierra Holanda y Amigos de la Tierra Nigeria, presentaron tres demandas contra Royal Dutch Shell PLC y Shell Development Company Petroleum de Nigeria ante el Tribunal de Distrito de La Haya, en los Países Bajos. Los demandantes solicitaban que el Tribunal declarase la responsabilidad civil extracontractual por los daños sufridos en sus propiedades y en el medio ambiente como consecuencia de los derrames de petróleo acaecidos en sus aldeas entre 2004 y 2006. Entre otros aspectos se solicita al Tribunal que ordene a las empresas la limpieza de los espacios contaminados y de las fuentes de agua, la aplicación de un plan para mantener las tuberías y prevenir los vertidos, y que establezca una indemnización para las víctimas.

En términos generales, los derrames que originan los daños tienen su origen en oleoductos o pozos petrolíferos operados o bajo licencia de las empresas demandadas, y responden a patrones similares en los tres casos. En particular, los demandantes atribuyen a la empresa negligencia en la manutención y vigilancia de los pozos y oleoductos, y alegan que las empresas demandadas no actuaron con celeridad para limitar sus efectos y no limpiaron correctamente las zonas afectadas. Asimismo, los demandantes invocaron la negligencia de la empresa matriz — Royal Dutch Shell PLC_ - al no asegurar que su filial llevase a cabo sus actividades en Nigeria de conformidad con los estándares técnicos más avanzados, a pesar de tener la capacidad para ello. 
Después de cuatro años, la tramitación del juicio culminó con la celebración de una audiencia conjunta el 11 de octubre de 2012. Las sentencias correspondientes a los tres casos fueron emitidas el pasado 30 de enero de $2013^{17}$. En síntesis, y con carácter general, el Tribunal de Distrito de La Haya exoneró de responsabilidad a la empresa matriz. Entiende el Tribunal que el derecho nigeriano, de aplicación a los hechos enjuiciados, no impone a las empresas ninguna obligación de velar por que sus subsidiarias no causen daños a terceros en el desarrollo de su actividad comercial.

Asimismo, exoneró de responsabilidad a la subsidiaria por los daños causados a raíz de dos de los tres derrames (en las aldeas de Goi y Oruma). En particular, el Tribunal consideró probado que estos derrames fueron causados por el sabotaje de oleoductos subterráneos. La dificultad en el acceso al oleoducto, que requería cavar una zanja de proporciones considerables, constituyó una de las principales circunstancias en las que el Tribunal se basó para determinar, de conformidad con el derecho nigeriano, que el sabotaje y los daños resultantes no fuesen imputables a una negligencia de SDCP en el deber de custodia y vigilancia de sus instalaciones. Por el contrario, la facilidad en el acceso, y la falta de un sellado apropiado del pozo petrolífero cuyo sabotaje causó el derrame en Ikot Ada Udo fue tomado en consideración por el Tribunal para declarar la responsabilidad civil extracontractual de la subsidiaria por incumplimiento negligente de su deber de custodia.

Lejos de poder calificarse de un éxito o fracaso rotundo de las pretensiones de las partes demandantes, la decisión del Tribunal de Distrito de La Haya constituye sin embargo un hito en la litigación transnacional contra empresas multinacionales por el hecho — que apenas cuenta con precedentes - de que un tribunal nacional del país en el que se ubica la empresa matriz se declare competente para conocer de reclamaciones de responsabilidad civil extracontractual por daños (ambientales) causados a terceros por empresas subsidiarias que operan en países terceros. Pese a la enorme dificultad que entraña este tipo de reclamaciones para las partes demandantes, ofrece una perspectiva de tutela judicial que hasta ahora se consideraba poco viable.

En efecto, el Código Procesal Civil neerlandés confiere competencia judicial internacional a los tribunales de dicho país para conocer de demandas de responsabilidad civil extracontractual dirigidas contra empresas domiciliadas en el extranjero, en litisconsorcio pasivo con empresas domiciliadas en Países Bajos ${ }^{18}$. En el contexto de los litigios que nos ocupan, las partes demandadas alegaron que los demandantes

ACCIONES

ENTRELAZADAS

contra daños

AMBIENTALES

GRAVES: EL

IMPACTO DE

SHELL EN NIGERIA

INTERTWINED

ACTIONS

AGAINST SERIOUS ENVIRONMENTAL

DAMAGE:THE

IMPACT OF SHELL

IN NIGERIA 
invocaron esta base competencial en abuso de derecho para poder demandar a una empresa extranjera en Holanda por hechos acaecidos en un tercer país. En particular, la empresa matriz alegó que la reclamación de su responsabilidad carecía a priori de cualquier fundamentación jurídica y, por tanto, de perspectivas procesales. No obstante, el Tribunal de Distrito de La Haya estableció que antes de entrar a conocer del fondo del asunto, las reclamaciones y los hechos planteados ofrecían un grado suficiente de plausibilidad sobre la expectativa de entrañar la responsabilidad de las empresas demandadas. Esta circunstancia se consideró suficiente para establecer la competencia judicial del tribunal neerlandés. El Tribunal también consideró que la ulterior desestimación de las pretensiones de los demandantes contra la empresa domiciliada en Países Bajos no hace decaer dicha competencia. En los términos comunes a las tres sentencias,

[...] the forum non conveniens restriction no longer plays any role in today's international private law. The District Court is of the opinion that the jurisdiction of the Dutch court in the matter against SPDC based on Section 7 DCCP does not cease to exist in the event that the claims against RDS were to be dismissed, not even if subsequently, in fact, no connection or hardly any connection would remain with Dutch jurisdiction ${ }^{19}$.

\section{II.2.2. Punto Nacional de Contacto de la OCDE}

Entre las vías a las que se ha recurrido para pedir responsabilidades a Shell figura también un marco de compromisos voluntarios como son las Líneas Directrices de la OCDE para Empresas Multinacionales, un conjunto de recomendaciones a las empresas multinacionales con el fin de promover un comportamiento responsable de las mismas ${ }^{20}$. Su cumplimiento está supervisado por el Punto Nacional de Contacto (PNC) de la OCDE en cada Estado miembro ${ }^{21}$. En el caso de Shell en Nigeria se ha recurrido también a la queja ante el Punto Nacional de Contacto (PNC) de la OCDE en los Países Bajos y en el Reino Unido,

19 Ver la sentencia del Tribunal de Distrito de La Haya (Sección Mercantil) de 30 de enero de 2013, asunto F.A. Akpan y Vereniging Milieudefensie c. Royal Dutch Shell PLC y Shell Petroleum Development Company of Nigeria Ltd. (C/09/337050 / HA ZA 09-1580), § 4.6.

20 Estas recomendaciones fueron adoptadas en 1976 y han sido revisadas en diversas ocasiones, la ultima en la reunión del Consejo de la OCDE a nivel ministerial de mayo 2011, (http://www.oecd.org/ daf/inv/mne/oecdguidelinesformultinationalenterprises.htm). Una traducción no oficial al español está disponible en: http://www.comercio.mineco.gob.es/es-ES/inversiones-exteriores/punto-nacionalcontacto-lineas-directrices/PDF/lineas-directrices-ocde-empresas-multinacionales/TraduccionDirectrices-y-Comentarios-2011.pdf.

21 Los PNCs deben contribuir a la resolución de los problemas que surjan en relación con la aplicación de las Directrices en casos específicos de manera imparcial, previsible, equitativa y compatible con los principios y normas de las Directrices. Para ello, deben realizar una evaluación inicial para determinar si las cuestiones planteadas merecen un examen más detenido y, si es así, deben ofrecer a las partes implicadas sus buenos oficios para facilitar la resolución de la diferencia, para lo cual deberán emprender una serie de consultas. Y al término de los procedimientos deben hacer públicos los resultados de los procedimientos, tanto si hay acuerdo como si no lo hay. 
por parte de Amigos de la Tierra Países Bajos, Amigos de la Tierra Internacional y Amnistía Internacional, el 25 de enero de 2011.

La denuncia contra Shell se centra en las repetidas declaraciones de la empresa en el sentido de que la mayoría de los derrames de petróleo en Nigeria, entre un 70 y un $85 \%$, se deben a sabotajes. Para los denunciantes se trata de una estrategia, tanto para eludir la responsabilidad y evitar tener que compensar a las víctimas, como para desviar la atención de su propia responsabilidad en el deterioro del delta del Níger y confundir a la opinión pública, a los consumidores y a sus propios accionistas. Para las organizaciones denunciantes tales declaraciones suponen la vulneración de las Líneas Directrices en tres aspectos:

La sección III, relativa a la publicación de informaciones, en la que se dispone que las empresas «deberán garantizar la revelación de información puntual, periódica, fiable y relevante acerca de sus actividades, sus estructuras, su situación financiera y sus resultados» y «se alienta a las empresas a aplicar unas normas de calidad rigurosas respecto a la información no financiera, incluida, en su caso, la elaboración de informes medioambientales y sociales».

La sección V, sobre medio ambiente, en la que se dice que «las empresas deberán tener debidamente en cuenta [...] la necesidad de proteger el medio ambiente y la salud y la seguridad públicas y de realizar, en general, sus actividades de una manera que contribuya al objetivo más amplio del desarrollo sostenible».

Y la sección VII, relativa a los intereses de los consumidores, en la que dispone que, en sus relaciones con los consumidores, «las empresas deberán actuar siguiendo unas prácticas comerciales, de marketing y publicitarias justas». Específicamente el punto 4 requiere «no realizar manifestaciones u omisiones ni participar en ninguna otra práctica que sea engañosa, equívoca, fraudulenta o desleal $»^{22}$.

Tras el examen inicial, el PNC de los Países Bajos notifico a los denunciantes, el 23 de febrero de 2011, que actuaría en nombre de los dos PNC y que estimaba que los hechos alegados merecían un examen más detenido, por lo que aceptó el caso. El PNC está llevando a cabo contactos para aproximar las posiciones de las partes.

\section{II.3. Reino Unido}

En agosto y diciembre de 2008 se produjeron dos graves vertidos casi consecutivos, por fugas debidas al deterioro de los oleoductos, que se

\section{ACCIONES}

ENTRELAZADAS

CONTRA DAÑOS

AMBIENTALES

GRAVES: EL

IMPACTO DE

SHELL EN NIGERIA

INTERTWINED

ACTIONS

AGAINST SERIOUS

ENVIRONMENTAL

DAMAGE: THE

IMPACT OF SHELL

IN NIGERIA 
prolongaron durante semanas, en las cercanías de Bodo, en Ogoniland ${ }^{23}$. Al no obtener justicia en Nigeria, en abril de 2011 se presentó ante la Corte Superior de Justicia del Reino Unido una demanda contra Royal Dutch Shell Plc. y su filial Shell Petroleum Development Company (Nigeria) Ltd., en representación de once mil habitantes de Bodo ${ }^{24}$. La filial de Shell aceptó su responsabilidad y reconoció la jurisdicción del Reino Unido. Pero las negociaciones abiertas entre las partes para acordar una compensación económica, que permitiera limpiar la zona, se rompieron y, en marzo de 2012, los representantes legales de los habitantes de Bodo presentaron una nueva demanda por daños y perjuicios contra Shell. El procedimiento sigue su curso.

\section{II.4. Estados Unidos}

$\mathrm{Al}$ amparo de la Alien Tort Claims Act ${ }^{25}$ se han presentado distintas demandas ante los tribunales civiles federales en Estados Unidos, en relación con la vulneración del derecho internacional por parte de la empresa Shell en Nigeria. Las más significativas son las relacionadas con los casos Wiwa y Kiobel.

\section{II.4.1. Wiwa v. Shell}

El 8 de noviembre de 1996, las empresas Royal Dutch Petroleum Company y Shell Transport and Trading Company fueron demandadas ante los tribunales federales por Ken Wiwa y otros nueve miembros del MOSOP. Los demandantes alegan que el gobierno militar nigeriano y las fuerzas de seguridad cometieron múltiples violaciones de los derechos humanos contra quienes llevaba a cabo una campaña no violenta contra el impacto ambiental causado por la extracción de petróleo en la región de Ogoni, en Nigeria, entre ellas, la ejecución, el 10 de noviembre de 1995, de varios de sus dirigentes, uno de ellos Ken Saro Wiwa, padre del primer demandante y presidente del MOSOP ${ }^{26}$.

Alegan también que la empresa Royal Dutch/Shell (nombre anterior de la empresa) fue cómplice en la comisión de estos abusos y, en particular, que las ejecuciones

23 Ver AMNISTÍA INTERNACIONAL La verdadera "tragedia». Demoras e inacción frente a los vertidos de petróleo del delta del Níger. Índice Al: AFR 44/018/2011, (http://www.amnistiainternacional.org/ publicacion-188), 2011.

24 Bodo Community v Shell Petroleum Development Company of Nigeria Case No. HQ11X01280.

25 Su breve texto dispone lo siguiente: «1350. Alien's action for tort. The district courts shall have original jurisdiction of any civil action by an alien for a tort only, committed in violation of the law of nations or a treaty of the United States" (Act of 24 September 1789, ch. 20, § 9 (b), 1 Stat. 79; June 25, 1948, ch. 646, § 1, 62 Stat. 934; 28 U.S.C. $§ 1350$ (2004)). Ver al respecto, PIGRAU, Antoni. "La responsabilidad civil de las empresas transnacionales a través de la Alien Tort Claims Act por su participación en violaciones de derechos humanos». Revista Española de Desarrollo y Cooperación, 25 (2010), pp. 113-130.

26 Fue nominado al Premio Nobel y le concedieron el Right Livelihood Award, en 1994, y el Premio Goldman, en 1995, por su defensa de los derechos humanos y el medio ambiente. 
were carried out with the knowledge, consent and/or support of defendants [...] and its agents and officers as part of a pattern of collaboration and/or conspiracy between Royal Dutch/Shell and the military junta of Nigeria to violently and ruthlessly suppress any opposition to Royal Dutch/Shell's conduct in its exploitation of oil and natural gas resources in Ogoni and in the Niger Delta.

La demanda fue rechazada el 25 de septiembre de 1998 por el juez Wood, del Tribunal del Distrito Sur de Nueva York, de acuerdo con la doctrina del forum non conveniens, por entender que el proceso debería desarrollarse en el Reino Unido, aunque no siguió las recomendaciones del informe previo de otro magistrado en el sentido de considerar la ausencia de jurisdicción personal sobre las empresas demandadas al considerar que no eran suficientemente relevantes sus vínculos comerciales con Estados Unidos. Los demandantes recurrieron la decisión en apelación y la Corte de Apelaciones del Segundo Circuito ${ }^{27}$ se pronunció el 14 de septiembre de 2000. En primer lugar, confirmó la apreciación del Tribunal de Distrito en cuanto a la existencia de jurisdicción personal sobre las empresas demandadas ${ }^{28}$. Pero, en cambio, decidió revocar la decisión del Tribunal de Distrito en relación con el forum non conveniens ${ }^{29}$.

Por tanto, el caso volvió al Tribunal de Distrito para que continuara con la tramitación del procedimiento abierto. Entre 2001 y 2009 fueron planteándose y resolviéndose distintos recursos ${ }^{30}$ que no sirvieron

27 En los Estados Unidos hay hasta 93 distritos judiciales divididos en 12 circuitos regionales, para cada uno de los cuales existe una Corte de Apelaciones. El Segundo Circuito corresponde a los Estados de Connecticut, Nueva York y Vermont.

28 "Under New York law, a foreign corporation is subject to general personal jurisdiction in New York if it is "doing business" in the state. [...] The continuous presence and substantial activities that satisfy the requirement of doing business do not necessarily need to be conducted by the foreign corporation itself. In certain circumstances, jurisdiction has been predicated upon activities performed in New York for a foreign corporation by an agent» (Wiwa v. Royal Dutch Petroleum Co., 226 F.3d 88, 95 (2d Cir. 2000)).

29 Y ello por tres motivos básicos: «In our view, the district court failed to give weight to three significant considerations that favor retaining jurisdiction for trial: (1) a United States resident plaintiff's choice of forum, (2) the interests of the United States in furnishing a forum to litigate claims of violations of the international standards of the law of human rights, and (3) the factors that led the district court to dismiss in favor [ $\left.{ }^{\star} 35\right]$ of a British forum were not particularly compelling. For the reasons developed below, we believe that they are outweighed by the considerations favoring exercise of the court's jurisdiction" (ibídem, 101).

30 Así, entre otras decisiones, el 5 de marzo de 2001 los demandantes incluyeron entre los demandados a Brian Anderson, responsable de las operaciones de Shell en Nigeria durante el periodo de tiempo relevante para el caso. Y la demanda fue de nuevo modificada el 16 de junio de 2003 . El 22 de febrero de 2002 el Tribunal de Distrito rechazo la aplicabilidad del Alien Tort Claims Act para algunos crímenes en relación con uno de los demandantes, pero la aceptó para los demás supuestos alegados (Wiwa v. Royal Dutch Petroleum Co., No 96-cv-8386, 2002 WL 319887, at 4 (S.D.N.Y., Fed. 28, 2002)). Y el 5 de abril de 2004 los demandantes incorporaron a la filial nigeriana Shell Petroleum Development Company de Nigeria (SPDC) como demandada. En noviembre de 2004 esta empresa presentó un recurso de inadmisión por falta de jurisdicción personal. El 4 de marzo de 2008 el Tribunal de Distrito aceptó el recurso. El 15 de abril de 2008 los demandantes apelaron la decisión en relación con el caso Wiwa (como se verá la misma cuestión se suscitó paralelamente en el caso Kiobel tras una decisión del 8 de marzo). El 3 de junio de 2009 la Corte de Apelación del Segundo Circuito anuló la decisión del Tribunal de Distrito y le remitió de nuevo el asunto. Entretanto, el 29 de septiembre de 2006 el Tribunal de Distrito aceptó, en el caso Kiobel, el recurso de los

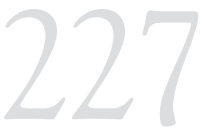

ACCIONES

ENTRELAZADAS CONTRA DAÑOS

AMBIENTALES

GRAVES: EL

IMPACTO DE

SHELL EN NIGERIA

INTERTWINED

ACTIONS

AGAINST SERIOUS ENVIRONMENTAL

DAMAGE:THE IMPACT OF SHELL IN NIGERIA 
para cerrar el procedimiento. Y cuando la celebración del juicio era inminente, los días 7 y 8 de junio de 2009 las partes formalizaron varios acuerdos para poner fin al litigio ${ }^{31}$. El acuerdo previó el pago de un total de 15,5 millones dólares (7,5 millones a cargo de Shell Petroleum N.V. y Shell Transport and Trading Company Ltd.; 3,5 a cargo de SPDC de Nigeria y 4,5 a cargo de Energy Equity Resources Limited), que cubren una compensación para los diez demandantes y una parte de los costos del proceso. El acuerdo también incluye el establecimiento de un fideicomiso en beneficio del pueblo ogoni, que será asignado a gestores independientes, con objeto de financiar distintas iniciativas de promoción social en su territorio.

\section{II.4.2. Kiobel v. Shell}

Esther Kiobel, la esposa del Dr. Barinem Kiobel, otro de los activistas ogoni ejecutados en 1995, presentó una demanda en septiembre de 2002 contra las mismas empresas. Alegaba que Shell, a través de su filial nigeriana Shell Petroleum Development Company de Nigeria (SPDC), facilitó el transporte de las tropas nigerianas, permitió que las propiedades de la empresa se utilizaran como base de operaciones para los ataques contra los ogoni, proporcionó alimentos a los soldados y les pagó. La demandante reclamaba, en consecuencia, que las empresas demandadas fueran condenadas como cómplices en la comisión de torturas, ejecuciones extrajudiciales y otras violaciones de conformidad con la ATCA.

El 29 de septiembre de 2006, tras distintos recursos previos de los acusados que fueron rechazados, el Tribunal del Distrito Sur de Nueva York acepta las alegaciones de tortura, detención ilegal y crímenes contra la humanidad y rechaza otras como la ejecución extrajudicial, crímenes contra los derechos a la vida, a la libertad o a la seguridad, el exilio forzado y la destrucción de propiedades como base material de aplicabilidad del ATCA al caso ${ }^{32}$.

Por otra parte, el 8 de marzo de 2008, el Tribunal de Distrito acepto la petición de los acusados de inadmisión del caso por falta de jurisdicción personal sobre las empresas ${ }^{33}$. Pero, tras el recurso de los demandantes, la ya citada decisión de 3 de junio de 2009 de la Corte de Apelación del Segundo Circuito que anuló la decisión del Tribunal de Distrito en el caso Wiwa lo lleva a reconsiderar el asunto en una nueva decisión de

mismos demandados en relación con los crímenes de ejecuciones extrajudiciales, el exilio forzado, crímenes contra los derechos a la vida, la libertad y la seguridad y la destrucción de la propiedad, pero lo rechazó, confirmando la viabilidad de la demanda, en relación con los crímenes contra la humanidad, la tortura y las detenciones arbitrarias (Kiobel v. Royal Dutch Petroleum Co., 456 F. Supp. 2d (S.D.N.Y. 2006)).

31 Pueden consultarse los documentos relativos a los acuerdos en la página web del Center for Constitutional Rights, (http://ccrjustice.org/ourcases/current-cases/wiwa-v.-royal-dutch-petroleum).

32 Kiobel v. Royal Dutch Petroleum Co., 456 F. Supp. 2d 457, 464-65, 467 (S.D.N.Y. 2006).

33 Kiobel v. Royal Dutch Petroleum Co., No. 02 Civ. 7618, 2008 WL 591869 (S.D.N.Y. 2008). 
16 de noviembre de 2009, pasando a denegar la causa de inadmisión alegada.

El 21 de junio de 2010, el Tribunal de Distrito consideró que SPDC era una empresa nigeriana con sede en Nigeria, que no tenía oficinas ni domicilio comercial en Estados Unidos y que los demandantes no habían demostrado que hubiera una relación comercial entre Estados Unidos y SPDC por lo que no era posible establecer la jurisdicción de los tribunales federales sobre la empresa, por lo que desestimó la demanda contra SPDC 34 .

Pero la decisión del Tribunal de Distrito de 2006, que estimaba parcialmente la viabilidad de las causas alegadas en vulneración del ATCA, fue apelada por los demandantes y, el 17 de septiembre de 2010, la Corte de Apelaciones del Segundo Circuito, en Nueva York, emitió una opinión sorprendente en relación con la posibilidad de demandar a las empresas en el marco del ATCA. La mayoría de la Corte confirmó la decisión del Tribunal de Distrito, pero además declaró que el ATCA no se podría utilizar para demandar a las empresas por violaciones del derecho internacional:

No corporation has ever been subject to any form of liability (whether civil, criminal, or otherwise) under the customary international law of human rights. Rather, sources of customary international law have, on several occasions, explicitly rejected the idea of corporate liability. Thus, corporate liability has not attained a discernable, much less universal, acceptance among nations of the world in their relations inter se, and it cannot, as a result, form the basis of a suit under the ATS ${ }^{35}$.

En opinión del juez José Cabranes, en nombre de la mayoría y aludiendo a un célebre pasaje de la sentencia del Tribunal de Núremberg:

From the beginning, however, the principle of individual liability for violations of international law has been limited to natural persons —not «juridical» persons such as corporations— because the moral responsibility for a crime so heinous and unbounded as to rise to the level of an «international crime» has rested solely with the individual men and women who have perpetrated $\mathrm{it}^{36}$.

34 Kiobel v. Royal Dutch Petroleum Company, No. 02 Civ. 7618, 2010 WL 2507025 (S.D.N.Y. June 21, 2010).

35 Kiobel v. Royal Dutch Petroleum Company, 621 F.3d 111, 149 (2d Cir. 2011), September 17, 2010.

$36 \mathrm{lbídem}$, p.7. En lo que se refiere a las demandas contra empresas en el marco del ATCA, la posición procesal que las mismas han venido expresando se resume en las ideas siguientes: no es posible demostrar, en el derecho internacional, la existencia de prohibiciones dirigidas a las empresas; en las pocas ocasiones en que el derecho internacional impone obligaciones a actores no estatales, no impone obligaciones o establece responsabilidades de personas jurídicas, como lo demuestra su exclusión de la jurisdicción de la Corte Penal Internacional; los conceptos de derecho nacional

ACCIONES

ENTRELAZADAS

CONTRA DAÑOS

AMBIENTALES

GRAVES: EL

IMPACTO DE

SHELL EN NIGERIA

INTERTWINED

ACTIONS

AGAINST SERIOUS

ENVIRONMENTAL

DAMAGE:THE

IMPACT OF SHELL

IN NIGERIA 

que comienza con el siguiente párrafo:

The majority opinion deals a substantial blow to international law and its undertaking to protect fundamental human rights. According to the rule my colleagues have created, one who earns profits by commercial exploitation of abuse of fundamental human rights can successfully shield those profits from victims' claims for compensation simply by taking the precaution of conducting the heinous operation in the corporate form. Without any support in either the precedents or the scholarship of international law, the majority takes the position that corporations, and other juridical entities, are not subject to international law, and for that reason such violators of fundamental human rights are free to retain any profits so earned without liability to their victims ${ }^{38}$.

El 14 de octubre de 2010, los demandantes presentaron una petición de nueva audiencia ante el panel de jueces y ante el plenario ante la Corte, peticiones que fueron rechazadas el 4 de febrero de 2011, por dos votos contra uno la primera y con un empate a cinco votos la segunda ${ }^{39}$. El 30 de septiembre de 2011 el Tribunal de Distrito decidió en consecuencia cerrar el caso.

como la conspiración o la complicidad no pueden ser empleados para ampliar la categoría de conductas prohibidas por el derecho internacional consuetudinario o la modalidad de participación en las mismas. Al respecto, ver por ejemplo las alegaciones de las empresas demandadas en In $\mathrm{Re}$ «Agent Orange» Product Liability Litigation, 373 F. Supp. 2d 7 (E.D.N.Y. 2005); o en Brief for the Chamber of Commerce of the United States o America As Amicus Curiae in Support of DefendantAppellee Talisman Energy, Inc., and in Support of Affirmance, May 8, 2007, en el litigio Presbyterian Church of Sudan v. Talisman Energy, Inc. No obstante, la condición de posibles demandadas de las empresas transnacionales de acuerdo con el ATCA, y por tanto su aptitud para ser responsables por violaciones del derecho internacional, aún con determinadas condiciones, ha sido ampliamente reconocida, desde Carmichael v. United Technologies Corp., 835 F.2d 109 (5th Cir. 1988), si bien la primera demanda efectivamente formulada contra una empresa fue la de 1996 contra UNOCAL, por su implicación en violaciones de derechos humanos en relación con la construcción del gasoducto de Yadana, en Myanmar. De particular interés resulta la decisión de la Corte del Distrito Este de Nueva York In Re «Agent Orange» Product Liability Litigation, 373 F. Supp. 2d 7, $56-57$ (E.D.N.Y. 2005). En general, ver StEPHENS, Beth. «Corporate Accountability: International Human Rights Litigation Against Corporations in US Courts». En KammingA, Menno \& Salman ZIA-ZARIFI (eds.). Liability of Multinational Corporations Under International Law. La Haya: Kluwer, 2000, pp. 209-229; PIGRAU, Antoni, «La responsabilidad de las empresas transnacionales por daños graves al medio ambiente: explorando la vía de la Alien Tort Claims Act» En BADIA, Anna \& otros (coords.). El derecho internacional ante los retos de nuestro tiempo. Homenaje a la Profesora Victoria Abellán Honrubia. Madrid/Barcelona/Buenos Aires: Marcial Pons, 2009, pp. 517-569.

37 En su opinión, «the pleadings do not assert facts which support a plausible assertion that Shell rendered assistance to the Nigerian military and police for the purpose of facilitating human rights abuses, as opposed to rendering such assistance for the purpose of obtaining protection for its petroleum operations with awareness that Nigerian forces would act abusively» (Kiobel v. Royal Dutch Petroleum Company, 621 F.3d 111, 149 (2d Cir. 2011), September 17, 2010).

38 Ibídem.

39 Kiobel v. Royal Dutch Petroleum Company, 642 F.3d 268 (2d Cir. 2011) y 642 F.3d 379 (2d Cir. 2011), February 4, 2011. 
En junio de 2011, los demandantes presentaron un recurso de apelación ante la Corte Suprema. El 17 de octubre de 2011 la Corte Suprema admitió el recurso a trámite, contra la posición de Shell expresada en su brief de 12 de agosto de 2011. La Corte debía pronunciarse sobre dos cuestiones:

1. Whether the issue of corporate civil tort liability under the Alien Tort Statute («ATS»), 28 U.S.C. $§ 1350$, is a merits question, as it has been treated by all courts prior to the decision below, or an issue of subject matter jurisdiction, as the court of appeals held for the first time.

2. Whether corporations are immune from tort liability for violations of the law of nations such as torture, extrajudicial executions or genocide, as the court of appeals decisions provides, or if corporations may be sued in the same manner as any other private party defendant under the ATS for such egregious violations, as the Eleventh Circuit has explicitly held.

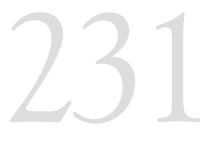

ACCIONES

ENTRELAZADAS

CONTRA DAÑOS

AMBIENTALES

GRAVES: EL

IMPACTO DE

SHELL EN NIGERIA

INTERTWINED

ACTIONS

AGAINST SERIOUS

ENVIRONMENTAL

DAMAGE:THE

IMPACT OF SHELL

IN NIGERIA

De hecho, el criterio de la Corte de Apelación del Segundo Circuito no ha sido seguido por otras Cortes que, con posterioridad a la decisión de la Corte de Apelaciones de Nueva York, se han pronunciado en sentido contrario afirmando la aplicabilidad del ATCA a las empresas ${ }^{40}$. La trascendencia de la decisión de la Corte Suprema para el futuro de la aplicabilidad del ATCA a las empresas es enorme ${ }^{41}$. Y las organizaciones de defensa de derechos humanos no ocultan su preocupación respecto de la decisión que pueda tomar una Corte compuesta mayoritariamente por magistrados conservadores.

La petición de certiorari de la parte demandante en el asunto Kiobel fue concedida por la Corte Suprema estadounidense el 17 de octubre de 2011. Después de la celebración de una primera audiencia, que tuvo lugar el 28 de febrero de 2012, el asunto fue remitido a una nueva audiencia en octubre del mismo año, para la cual las partes fueron emplazadas a presentar escritos complementarios que abordasen la cuestión «whether and under what circumstances the Alien Tort Statute $[\ldots]$ allows courts to recognize a cause of action for violations of the law of nations occurring within the territory of a sovereign other than the United States» ${ }^{42}$.

40 Es el caso de la Corte de Apelaciones del Noveno Circuito - Sarei v. Rio Tinto, PLC, No. 02-56256, 2011 WL 5041927 (9th Cir. Oct. 25, 2011) (en banc) -, de la Corte de Apelaciones del Distrito de Columbia -Doe v. Exxon Mobil Corp., 2011 U.S. App. LEXIS 13934 (D.C. Cir. July 8, 2011) - y de la Corte de Apelaciones del Séptimo Circuito - en Flomo v. Firestone Natural Rubber Co., LLC, 643 F.3d 1013 (7th Cir. Ind. 2011)-.

41 La Corte Suprema solamente se ha pronunciado hasta ahora en una ocasión en relación con el ATCA, en el caso Álvarez-Machain, el 29 de junio de 2004. Ver Sosa v. Alvarez-Machain, 542 U.S. 692 (2004), 124 S.Ct. 2739 (2004).

42 Ver http://www.supremecourt.gov/qp/10-01491qp.pdf. 
Resulta evidente que la decisión de la Corte Suprema — que se espera para finales del primer trimestre de 2013 - será determinante para el futuro de la aplicabilidad del ATCA a las empresas y, en general, para enjuiciar violaciones de normas internacionales cometidas en el extranjero. La importancia de esta decisión también se hace evidente por el ingente número de informes de amicus curiae sometidos a la Corte Suprema en relación con este caso ${ }^{43}$, entre los que merece la pena destacar especialmente los dos informes presentados por la Administración de los EE.UU. Mientras el primer informe, que aborda la cuestión de la posible inmunidad de las corporaciones de responsabilidad extracontractual por violaciones de normas internacionales, apoya los peticionarios ${ }^{44}$, en el escrito complementario sobre la admisibilidad de las denuncias extraterritoriales, la Administración responde a la pregunta de manera afirmativa, como cuestión de principio, pero considera que las alegaciones de los demandantes no cumplen los requisitos necesarios y pide una aplicación reforzada de las doctrinas del agotamiento previo de los recursos y del forum non conveniens para las reclamaciones basadas en el ATCA ${ }^{45}$.

\section{ACCIONES EMPRENDIDAS ANTE INSTITUCIONES INTERNACIONALES}

\section{III.1.Comisión Africana de Derechos Humanos y de los Pueblos}

La actividad de Shell en Nigeria también fue llevada al seno del sistema africano de protección de los derechos humanos.

La Comisión Africana de Derechos Humanos y de los Pueblos adoptó una decisión, en octubre de $2001^{46}$, en la que consideró que Nigeria había violado los artículos 2, 4, 14, 16, 18(1), 21 y 24 de la Carta Africana de Derechos Humanos y de los Pueblos ${ }^{47}$, en relación con el

43 Ver http://www.supremecourt.gov/Search.aspx?FileName=/docketfiles/10-1491.htm.

44 Entre otros argumentos se expone el siguiente: «Courts may recognize corporate liability in actions under the ATS as a matter of federal common law. The text and history of the ATS itself provide no basis for distinguishing between natural and juridical persons. Corporations have been subject to suit for centuries, and the concept of corporate liability is a well-settled part of our 'legal culture'» (Brief for the United States as Amicus Curiae Supporting Petitioner, Supreme Court, Esther Kiobel, individually and on behalf of her late husband Dr. Barinem Kiobel, et al., v Royal Dutch Petroleum Co., et al, 21 December 2011, [7]-[8], (www.earthrights.org/sites/default/files/documents/USG-Kiobel-amicus.pdf)).

45 Ver Supplemental Brief for the United States as Amicus Curiae in Partial Support of Affirmance, US Supreme Court, Esther Kiobel, individually and on behalf of her late husband Dr. Barinem Kiobel, et al., v Royal Dutch Petroleum Co., et al, 13 June 2012, (http://sblog.s3.amazonaws.com/wp-content/ uploads/2012/06/Kiobel-US-supp-brief-6-13-12.pdf).

46 The Social and Economic Rights Action Center and the Center for Economic and Social Rights v. Nigeria, African Commission on Human and Peoples' Rights, Comm. No. 155/96 (2001). Ver al respecto, SHELTON, Dinah. «International Decisions: Decision Regarding Communication 155/96 (Social and Economic Rights Action Center / Center for Economic and Social Rights v. Nigeria). Case No. ACHPR/COMM/A044/1». En American Journal of International Law, 96 (2002), pp. 937-942.

47 African [Banjul] Charter on Human and Peoples' Rights, June 27, 1981, OAU Doc. CAB/LEG/67/3 rev. 5; 21 I.L.M. 58 (1982). 
pueblo ogoni, violaciones en las que considera implicadas a las empresas Nigerian National Petroleum Company (NNPC), en consorcio con Shell Petroleum Development Corporation (SPDC).

En relación con el derecho a un medio ambiente satisfactorio o sano, reconocido en el artículo 24 de la Carta ${ }^{48}$, la Comisión consideró que:

It requires the State to take reasonable and other measures to prevent pollution and ecological degradation, to promote conservation, and to secure an ecologically sustainable development and use of natural resources. [...] Undoubtedly and admittedly, the government of Nigeria, through NNPC has the right to produce oil, the income from which will be used to fulfil the economic and social rights of Nigerians. But the care that should have been taken as outlined in the preceding paragraph and which would have protected the rights of the victims of the violations complained of was not taken ${ }^{49}$.

En cuanto al artículo 21, relativo a la soberanía sobre los recursos naturales ${ }^{50}$, la Comisión señala las obligaciones de Nigeria en relación con las empresas que operan en el país:

Governments have a duty to protect their citizens, not only through appropriate legislation and effective enforcement but also by protecting them from damaging acts that may be perpetrated by private parties [...]. The Commission notes that in the present case, despite its obligation to protect persons against interferences in the enjoyment of their rights, the Government of Nigeria facilitated the destruction of the Ogoniland. Contrary to its Charter obligations and despite such internationally established principles, the Nigerian Government has given the green light to private actors, and the oil Companies in particular, to devastatingly affect the well-being of the Ogonis. By any measure of standards, its practice falls short of the minimum conduct expected of governments, and therefore, is in violation of Article 21 of the African Charter $^{51}$.

48 Artículo 24: «All peoples shall have the right to a general satisfactory environment favourable to their development.» También el Artículo 16 dispone que: «(1) Every individual shall have the right to enjoy the best attainable state of physical and mental health. (2) States Parties to the present Charter shall take the necessary measures to protect the health of their people and to ensure that they receive medical attention when they are sick».

49 Párrafos 52-54.

50 Artículo 21: «1. All peoples shall freely dispose of their wealth and natural resources. This right shall be exercised in the exclusive interest of the people. In no case shall a people be deprived of it».

51 Párrafos 57-58.

ACCIONES

ENTRELAZADAS CONTRA DAÑOS

AMBIENTALES

GRAVES: EL

IMPACTO DE

SHELL EN NIGERIA

INTERTWINED

ACTIONS

AGAINST SERIOUS

ENVIRONMENTAL

DAMAGE:THE

IMPACT OF SHELL

IN NIGERIA 
También conecta los daños al medio ambiente con el derecho a la alimentación, implícito en el derecho a la salud:

66. The government's treatment of the Ogonis has violated all three minimum duties of the right to food. The government has destroyed food sources through its security forces and State Oil Company; has allowed private oil companies to destroy food sources; and, through terror, has created significant obstacles to Ogoni communities trying to feed themselves. The Nigerian government has again fallen short of what is expected of it as under the provisions of the African Charter and international human rights standards, and hence, is in violation of the right to food of the Ogonis ${ }^{52}$.

Finalmente, en relación con el derecho a la vida, enunciado en el artículo 4 de la $\mathrm{Carta}^{53}$, se afirma lo siguiente:

Given the wide spread violations perpetrated by the Government of Nigeria and by private actors (be it following its clear blessing or not), the most fundamental of all human rights, the right to life has been violated. The Security forces were given the green light to decisively deal with the Ogonis, which was illustrated by the wide spread terrorisations and killings. The pollution and environmental degradation to a level humanly unacceptable has made it living in the Ogoni land a nightmare. The survival of the Ogonis depended on their land and farms that were destroyed by the direct involvement of the Government. These and similar brutalities not only persecuted individuals in Ogoniland but also the whole of the Ogoni Community as a whole. They affected the life of the Ogoni Society as a whole ${ }^{54}$.

La Comisión, en sus recomendaciones, entre otras, insta al Gobierno de Nigeria a perseguir a los responsables de las fuerzas de seguridad de la Nigerian National Petroleum Company y de otras instituciones relevantes implicadas en violaciones de derechos humanos.

\section{III.2. Corte de Justicia de la Comunidad Económica de Estados de África Occidental ${ }^{55}$}

La Corte de Justicia de la Comunidad ECOWAS fue creada por un protocolo firmado en Abuja, el 6 de julio de $1991^{56}$, y fue posteriormente

\footnotetext{
52 Párrafos 65-66.

53 Artículo 4: «Human beings are inviolable. Every human being shall be entitled to respect for his life and the integrity of his person. No one may be arbitrarily deprived of this right".

55 La Comunidad Económica de Estados de África Occidental (CEDEAO/ECOWAS) es una organización que agrupa a quince países de África Occidental, creada el 28 de mayo de 1975, mediante el Tratado de Lagos, con el objetivo de promover la integración económica de la región.

56 Protocol A/P.I/7/91 on the Community Court of Justice, (http://www.courtecowas.org/site2012/pdf_ files/protocol.pdf).
} 
incluido en el artículo 6 del Tratado Revisado de la Comunidad, adoptado en Cotonou el 24 de julio de $1993^{57}$. No obstante, la Corte no comenzó oficialmente a funcionar hasta que el protocolo entró en vigor en 1996 y, en la práctica, en 2001. Entre otras, la Corte tiene competencia para juzgar vulneraciones de los derechos humanos. Desde $2005^{58}$, los ciudadanos de los Estados miembros de la Comunidad y también las empresas pueden presentar quejas por violaciones de derechos humanos por parte de actores estatales ante la Corte, sin exigirse el previo agotamiento de los recursos internos.

El 24 de julio de 2009, la organización no gubernamental nigeriana Socio-Economic Rights and Accountability Project (SERAP) presentó una demanda por presunta violación de los derechos humanos en la región del delta del Níger, contra el presidente de la República de Nigeria, el fiscal general, la empresa pública Nigerian National Petroleum Corporation (NNPC), que es el accionista mayoritario en todos los joint ventures, la filial de Royal Dutch Shell, Shell Petroleum Development Company (SPDC), Elf Petroleum Nigeria Ltd., Agip Nigeria Plc., Chevron Nigeria Oil Plc. y ExxonMobil Corporation.

El interés de la demanda radica en que no se refiere a un episodio concreto sino al conjunto de la explotación petrolera en el delta del Níger. Se denuncia, por una parte, la violación del derecho a un nivel de vida adecuado, incluido el derecho a la alimentación, al trabajo, a la salud, al agua, a la vida y a la dignidad humana, a un medio ambiente limpio y saludable, y al el desarrollo económico y social, como consecuencia de la repercusión de la contaminación relacionada con el petróleo; y, por otra, la incapacidad de hacer cumplir las leyes y reglamentos para proteger el medio ambiente y prevenir la contaminación, con lo que el gobierno de Nigeria ha agravado el problema, creando una cultura de la impunidad para las compañías petroleras en el delta del Níger. Igualmente se alega que las violaciones de los derechos humanos por parte de las empresas a menudo son consecuencia de la falta de la debida diligencia y planificación adecuada o de acciones deliberadas.

En su decisión de 10 de diciembre de 201059, la Corte aborda las objeciones preliminares presentadas por las empresas demandadas, que alegan, entre otros motivos para solicitar la inadmisión de la demanda,

57 Revised Treaty of the Economic Community of West African States (ECOWAS), 24 July 1993, (http:// www.unhcr.org/refworld/docid/492182d92.html).

58 Mediante un nuevo Protocolo adoptado en Accra el 19 de enero de 2005, que añade un nuevo Artículo 10: Supplementary Protocol A/SP.1/01/05 Amending the Preamble and Articles 1, 2, 9 and 30 of Protocol A/P.I/7/91 relating to the Community Court of Justice and Article 4 Paragraph 1 of the said Protocol, (http://www.courtecowas.org/site2012/pdf_files/supplementary_protocol.pdf).

59 SERAP v. Nigeria, Ruling, Suit No: ECW/CCJ/APP/08/09 and RUL. No: ECW/CCJ/APP/07/10 (ECOWAS, Dec. 10, 2010), (http://www.worldcourts.com/ecowasccj/eng/decisions/2010.12.10_ SERAP_v_Nigeria.htm).

ACCIONES

ENTRELAZADAS

conTRA DAÑos

AMBIENTALES

GRAVES: EL

IMPACTO DE

SHELL EN NIGERIA

INTERTWINED

ACTIONS

AGAINST SERIOUS

ENVIRONMENTAL

DAMAGE:THE

IMPACT OF SHELL

IN NIGERIA 
la falta de legitimación de los demandantes y la falta de competencia de la Corte en relación con ellas.

La Corte recuerda que debe aplicar la Carta Africana de Derechos Humanos y de los Pueblos que reconoce, entre otros, el derecho de los pueblos a su desarrollo económico, social y cultural, y el derecho de los pueblos a un medio ambiente satisfactorio y favorable para su desarrollo, para señalar que, conforme al derecho internacional de los derechos humanos, «las personas cuyos derechos hayan sido violados deben tener acceso a un recurso efectivo» y que los Estados deben tomar medidas para proteger los derechos económicos, sociales y culturales de los pueblos de las acciones de los actores no estatales que podrían socavar el disfrute de esos derechos. En su decisión la Corte confirma la legitimación de la ONG demandante, aludiendo a las disposiciones del Convenio de Aarhus sobre el acceso a la información, la participación del público en el proceso de toma de decisiones y el acceso a la justicia en materia de medio ambiente de $1989^{60}$, de la Convención Americana de Derechos Humanos de 1969, y del Reglamento de Procedimiento de la Corte Africana de Justicia y Derechos Humanos. Y concluye así:

[...] the Court holds that an NGO duly constituted according to national law of any ECOWAS Member State, and enjoying observer status before ECOWAS institutions, can file complaints against Human Rights violation in case that the victim is not just a single individual, but a large group of individuals or even entire communities ${ }^{61}$.

La otra cuestión de fondo planteada es la de la responsabilidad de las empresas. Al respecto la Corte señala lo siguiente:

In fact, one of the paradoxes that characterize International Law presently is the fact that States and individuals can be held accountable internationally, while companies cannot ${ }^{62}$.

Y considera que esta situación, que califica de anomalía, no ha evolucionado:

Despite the campaigned launched advocacy organizations towards new developments, the bare truth, however, is that the process of codification of international Law has not yet arrived at a point that allows the claim against corporations to be brought before

\footnotetext{
60 «Although the convention is not a binding instrument on African States, its importance, as a persuasive evidence of an international communis opinion juris in allowing NGOs to access the Courts for protection of Human Rights related to the environment, cannot be ignored or underestimated by this court» (ibídem, párrafo 58).

61 lbídem, párrafo 61.

62 Ibídem, párrafo 65.
} 
International Courts. Any attempts to do so have been dismissed on the basis that the Companies are not parties to the treaties that the international courts are empowered to enforce. This understanding is widely shared among regional courts with jurisdiction over Human Rights ${ }^{63}$.

Por lo que, aludiendo a una decisión anterior ${ }^{64}$, la Corte confirma que solamente los Estados miembros y las instituciones de la Comunidad Económica pueden ser demandadas ante ella por violaciones de derechos humanos, como consecuencia de ello carece de jurisdicción sobre las empresas demandadas.

En consecuencia SERAP presentó una nueva demanda contra el presidente y el fiscal general de Nigeria el 11 de marzo de 2011, en términos similares a la anterior, acompañándolos de datos relativos a distintos accidentes concretos, por violación de numerosos artículos de la Carta Africana de Derechos Humanos y de los Pueblos, del Pacto Internacional de Derechos Civiles y Politicos y del Pacto Internacional de Derechos Económicos, Sociales y Culturales y del artículo 15 de la Declaración Universal de Derechos Humanos.

Los demandados presentaron nuevas objeciones, entre ellas las relativas a la legitimación de los demandantes, a la falta de jurisdicción de la Corte en relación con los pactos internacionales de derechos humanos o a la prescripción de ciertos hechos denunciados. Todas ellas fueron rechazadas por la Corte en su Sentencia de 14 de diciembre de $2012^{65}$.

La Corte parte de la constatación de la larga duración de la exploración y la explotación petrolífera en la región que ha causado y continua causando el deterioro del suelo y del agua, y que los vertidos de petróleo debidos a la corrosión de las tuberías o a actos de vandalismo constituyen la primera causa de contaminación, hechos que han sido admitidos por el Gobierno de Nigeria. Considera que los vertidos destruyen la vida acuática y la fertilidad del suelo con efectos adversos para la salud y los medios de vida de las personas que viven en la región. Por tanto, si hay acuerdo en la existencia de vertidos, ello implica que hay contaminación.

63 Ibídem, párrafo 69.

64 «From what has been said, the conclusion to be drawn is that for the dispute between individuals on alleged violation of Human Rights as enshrined in the African Charters on Human and Peoples' Rights, the natural and proper venue before which the case may be pleaded is the domestic court of the State party where the violation occurred is only when at the national level, there is no appropriate and effective forum for seeking redress against individuals, that the victim of such offences may bring an action before an international court, not against the individuals, rather against the signatory State for failure to ensure the protection and respect for the Human Rights allegedly violated" (Peter David v. Ambassador Ralph Uwechue, 11 June 2010; citada en ibídem, párrafo 72).

65 The Court of Justice of the Economic Community of West African States (ECOWAS): SERAP, Applicant v. Federal Republic of Nigeria, Defendant, General List NECW/CCJ/APP/08/09. Judgment $\mathrm{N}^{\circ}$ ECW/CCJ/JUD/18/12, 14 December 2012, (http://www.courtecowas.org/site2012/pdf_files/ decisions/judgements/2012/SERAP_V_FEDERAL_REPUBLIC_OF_NIGERIA.pdf). 
Según la Corte, incumbe al Gobierno de Nigeria prevenir o remediar la situación, exigir responsabilidades a quienes han causado la situación y garantizar la adecuada reparación a las víctimas, en virtud del derecho a un medio ambiente satisfactorio reconocido en el artículo 24 de la Carta Africana de Derechos Humanos y de los Pueblos, en relación con el artículo 1 de la misma que dispone que los Estados miembros deben reconocer los derechos incluidos en la Carta y deben adoptar las medidas legislativas o de otro tipo necesarias para hacerlos efectivos.

Según la Corte:

This means that the adoption of the legislation, no matter how advanced it may be, or the creation of agencies inspired by the world's best models, as well as the allocation of financial resources in equitable amounts, may still fall short of compliance with international obligations in matters of environmental protection if these measures just remain on paper and are not accompanied by additional and concrete measures aimed at preventing the occurrence of damage or ensuring accountability, with the effective reparation of the environmental damage suffered ${ }^{66}$.

En opinión de la Corte, el problema principal reside en la falta de ejecutoriedad de la legislación vigente por parte de las autoridades de Nigeria encargadas de la supervisión de la industria petrolífera ${ }^{67}$.

En consecuencia la Corte concluye que Nigeria ha incumplido sus obligaciones en términos de vigilancia y diligencia y ha violado los artículos 1 y 24 de la Carta, en un razonamiento concordante con el ya mencionado de la Comisión Africana de Derechos Humanos y de los Pueblos.

No obstante, apartándose de la jurisprudencia de otros tribunales como, por ejemplo, la Corte Interamericana de Derechos Humanos, no considera procedente otorgar una indemnización económica a las víctimas y prefiere buscar otro tipo de beneficio colectivo, por lo que ordena al Gobierno de Nigeria que:

66 Ibídem, párrafo 105

67 Ver ibídem, párrafo 109. Y añade: «lt is significant to note that despite all the laws it has adopted and all the agencies it has created, the Federal Republic of Nigeria was not able to point out in its pleadings a single action that has been taken in recent years to seriously and diligently hold accountable any of the perpetrators of the many acts of environmental degradation which occurred in the Niger Delta Region» (ibídem, párrafo 110).

"And it is precisely this omission to act, to prevent damage to the environment and to make accountable the offenders, who feel free to carry on their harmful activities, with clear expectation of impunity, that characterises the violation by the Federal Republic of Nigeria of its international obligations under Articles 1 and 24 of the African Charter on Human and Peoples'Rights» (ibídem, párrafo 111). 
[...] adopte todas las medidas efectivas, en el más breve espacio de tiempo posible, para asegurar la restauración del medio ambiente del Delta del Níger; adopte todas las medidas necesarias para prevenir los daños al medio ambiente; adopte todas las medidas para hacer responsables a los perpetradores del daño ambiental ${ }^{68}$

\section{CONCLUSIONES}

Shell es para Nigeria una herencia directa del colonialismo. Obtuvo un permiso de exploración en Nigeria en 1938; descubrió el primer campo de petróleo con interés comercial en 1956 e inició las exportaciones de petróleo en el delta del Níger en 1958. Nigeria accedió a la independencia en 1960.

El impacto negativo de las operaciones de Shell en Nigeria ha sido de una dimensión extraordinaria. Se han dañado bienes comunes como el aire, el suelo o el agua; se ha emitido una gran cantidad de gases de efecto invernadero y se ha afectado la biodiversidad. Pero también se han causado daños a las personas especialmente en forma de afectaciones graves a la salud y a sus formas de subsistencia tradicional, la agricultura y la pesca; y en conexión con su oposición a las actividades petroleras se han vulnerado muchos otros derechos civiles y políticos, incluido el derecho a la vida.

Las víctimas han podido constatar la distancia que hay entre la existencia de leyes escritas y su aplicación efectiva, así como la enorme influencia que las grandes empresas transnacionales petroleras que operan en Nigeria pueden ejercer sobre las estructuras del Estado con la finalidad de eludir, en la mayor parte de los casos, sus responsabilidades por los daños causados.

Por eso, ante el escenario de una predominante carencia de acceso real a la justicia en Nigeria y teniendo en cuenta la inexistencia de vías internacionales para reclamar la responsabilidad directamente a las empresas, las víctimas han buscado otras vías para hacer efectiva la responsabilidad de Shell. Por una parte, a través de las posibilidades de la jurisdicción extraterritorial de otros tribunales nacionales: de manera directa en los Países Bajos y el Reino Unido y, mediante la conexión con la vulneración grave de derechos humanos, en los Estados Unidos. Por otra parte, ante mecanismos internacionales de protección de los derechos humanos en el plano regional (Comisión Africana de Derechos Humanos y de los Pueblos) o subregional (Corte de Justicia de la Comunidad Económica de Estados de África Occidental), al amparo de un texto jurídico como es la Carta Africana de Derechos Humanos y de los Pueblos que también incluye, de manera específica, el derecho al

\section{ACCIONES}

ENTRELAZADAS

CONTRA DAÑOS

AMBIENTALES

GRAVES: EL

IMPACTO DE

SHELL EN NIGERIA

INTERTWINED

ACTIONS

AGAINST SERIOUS

ENVIRONMENTAL

DAMAGE: THE

IMPACT OF SHELL

IN NIGERIA 
medio ambiente. Incluso han recurrido a mecanismos de supervisión de códigos de responsabilidad social de asunción voluntaria como las Líneas Directrices de la OCDE para empresas multinacionales.

Y en ese camino, mediante esas acciones entrelazadas, con todo el sacrificio y el desgaste humano y económico que comportan litigios que se alargan durante años en lugares lejanos, y con todas las limitaciones de las distintas vías empleadas, han podido hacer visibles algunas pequeñas grietas en un sistema jurídico internacional diseñado para facilitar las operaciones de las grandes empresas multinacionales y en el que pueden casi siempre hacer valer su poder y su dinero para blindarse frente a cualquier responsabilidad por los daños que causan.

Y esas acciones han forzado a Shell a negociar en Estados Unidos para evitar un juicio en el caso Wiwa; han obtenido una sentencia favorable en uno de los casos planteados en los Países Bajos, por responsabilidad extracontractual de una empresa nigeriana por daños causados en Nigeria, y pueden obtener otra en el Reino Unido; han obtenido decisiones favorables en la Comisión Africana de Derechos Humanos y de los Pueblos y, mucho más relevante, una sentencia de la Corte de Justicia de la Comunidad Económica de Estados de África Occidental que ordena a Nigeria hacer justicia y castigar a los responsables de la contaminación en el delta del Níger. Incluso han sido el detonante de que el alcance de la propia jurisdicción extraterritorial de los tribunales federales estadounidenses en el marco del ATCA esté en tela de juicio ante la misma Corte Suprema.

Aunque diversas, esas acciones han acabado por alimentarse mutuamente y han multiplicado la visibilidad del conflicto hasta hacerlo políticamente insoportable en el plano internacional, como lo prueba el informe de impacto emitido por el propio Programa de las Naciones Unidas para el Medio Ambiente. El caso de Shell no es único y en otros casos similares se constata esta práctica de acciones entrelazadas, pero es enormemente representativo. 